\title{
Glomerular Filtration Rate from B-2 Microglobulin Adjusted for BSA Measurement
}

National Cancer Institute

\section{Source}

National Cancer Institute. Glomerular Filtration Rate from B-2 Microglobulin Adjusted for BSA Measurement. NCI Thesaurus. Code C100450.

A measurement of the glomerular filtration rate (GFR) based on the clearance of beta-2 microg lobulin after adjusting it for the body surface area. 